\title{
Herding and stochastic volatility
}

\author{
Walter FARKAS ${ }^{1,3}$ Ciprian NeCUlA ${ }^{\ddagger 1,2} \quad$ Boris WAELCHLI $^{1}$
}

First version: 30 September 2015

This version: 30 October 2015

\begin{abstract}
In this paper we develop a one-factor non-affine stochastic volatility option pricing model where the dynamics of the underlying is endogenously determined from micro-foundations. The interaction and herding of the agents trading the underlying asset induce an amplification of the volatility of the asset over the volatility of the fundamentals. Although the model is non-affine, a closed form option pricing formula can still be derived by using a Gauss-Hermite series expansion methodology. The model is calibrated using S\&P 500 index options for the period 1996-2013. When its results are compared to some benchmark models we find that the new non-affine one-factor model outperforms the affine one-factor Heston model and it is competitive, especially out-of-sample, with the affine two-factor double Heston model.
\end{abstract}

Keywords: herding, non-affine option pricing model, Gauss-Hermite expansion JEL Classification: G12, C61

Acknowledgements: We are grateful to Gabriel Drimus for helpful comments. The research leading to these results has received funding from the SCIEX Project 11.159 and from the European Union Seventh Framework Programme (FP7/2007-2013) under the MC-IEF grant agreement n. 627701.

\footnotetext{
${ }^{1}$ Department of Banking and Finance, University of Zurich, Plattenstrasse 14, CH-8032, Zurich, Switzerland

${ }^{2}$ Department of Money and Banking, DOFIN, Bucharest University of Economic Studies, Bucharest, Romania

${ }^{3}$ Department of Mathematics, ETH Zurich, Raemistrasse 101, CH-8092 Zurich, Switzerland

${ }^{\ddagger}$ Corresponding author, email: ciprian.necula@bf.uzh.ch
} 


\section{Introduction}

The Black and Scholes (1973) model for option pricing is a cornerstone of the modern financial theory. However, there is an extensive empirical literature that has documented the biases of this classical model. The most striking of these biases is known as the "volatility smile" and refers to the stylized fact that observed market prices for out-of-the-money put prices and in-the-money call prices are higher than the theoretical prices obtained by the Black-Scholes model. One popular approach for taking into account the "volatility smile" is the use of exogenous stochastic volatility models that allow for negative correlation between the stock return and its instantaneous variance (Heston, 1993). In this type of models one departs from the central hypothesis of the Black-Scholes model concerning a constant level of the volatility, by specifying exogenously a dynamics for the instantaneous variance of the financial asset under study. The negative correlation between stock returns and the instantaneous volatility captures another stylized fact, the so called "leverage effect": decreases in the stock price are associated with larger increases in volatility than similar increases in the price (Black, 1976). Of course, exogenous stochastic volatility models cannot explain the mechanisms responsible for inducing and amplifying the asset price volatility. Moreover, a vast majority of these exogenous continuous time stochastic volatility models are affine: the drift term and squared diffusion term in the stochastic differential equation that describe the dynamics of the instantaneous variance are linear. The drift term captures, using a linear function, the fact that volatility oscillates around a long-run level, in a mean-reverting fashion.

A large literature on affine stochastic volatility models has emerged focusing on improving the path-breaking model of Heston (1993): models that allow for jumps in the dynamics of the price of the financial asset, in order to account for large moves such in the case of crashes (Bates, 1996; Bakshi, Cao and Chen, 1997), models 
allowing that the long-run variance is itself a stochastic process, modeled as a diffusion process or as a discrete state Markov process (Bardgett, Gourier and Leippold, 2013; Kaeck and Alexander, 2012), models that allow for jumps in the dynamics of the variance (Eraker, 2004; Broadie, Chernov and Johannes, 2007), two-factor models that generates stochastic correlation between returns and volatility (Christoffersen, Heston and Jacobs, 2009), or three-factor models with jumps both in the dynamics of the underlying and of the volatility (Andersen, Fusari and Todorov, 2015).

The results of a series of empirical studies such as Pan (2002), Jones (2003), Duan and Yeh (2010) suggest that there still is a need for improving affine stochastic volatility models. Further support for non-affine stochastic volatility models is provided by Kaeck and Alexander (2012) which present comprehensive empirical results regarding the option pricing performance of affine and non-affine continuous time stochastic volatility models. The authors consider an exogenous non-affine constant elasticity of volatility (CEV)-type stochastic volatility model augmented by jumps in both the price and variance process and by a stochastic long-run variance level. They find out that non-affine diffusion models clearly out-perform their affine counterparts both in-sample and out-of-sample. Moreover, they point out that the inclusion of jumps in the model is less important than allowing for non-affine dynamics. Similarly Drimus (2012) shows that the Heston related non-affine stochastic 3/2 model gives prices of options on realized variance which allow upward sloping implied volatility of variance smiles in contrast to the affine Heston model.

The purpose of the paper is to develop an endogenous and non-affine stochastic volatility model where the volatility induced by fundamentals is amplified due to the interaction and herding of the agents involved in trading a financial asset. At the same time, we show how one can price European options in the context of the model using the closed form formula based on the Gauss-Hermite series expansion methodology 
(Necula, Drimus and Farkas, 2013). We estimate the parameters of the model by a combination of non-linear optimization techniques using S\&P 500 index options, and conduct a study about the performance of the new option pricing model.

The paper is structured in six sections. In the second section we discuss the microfoundations and derive the dynamics equation of the asset price in the continuous time limit. In the third section we outline a method for computing option prices in the context of the model. The fourth section presents the data set and the estimation methodology. In the fifth section we report the result of the estimation and of the empirical investigation of the option pricing performance of the model. The final section concludes. The appendix collects the proofs of the propositions.

\section{The dynamics of the underlying}

We consider a financial market model with $N$ economic agents trading a single risky asset. For now, we assume that time is discrete denoted by $t_{k}$ with $t_{k+1}-t_{k}=1 / n$ and, later, we will determine the continuous time limit $(n \rightarrow \infty)$. As usual in the literature on micro-foundations of diffusions models for asset prices (Follmer and Schweizer, 1993; Follmer, Horst and Kirman, 2005; Horst, 2005), we do not formulate an individual optimization problem, but, specify directly the excess demand function for the risky asset of each individual agent. More specifically, the excess demand function of an agent $a$ at time $t_{k+1},{ }^{a} e_{k+1}^{n}(p)$ is given by

$$
{ }^{a} e_{k+1}^{n}(p)=\frac{{ }^{a} \hat{S}_{k+1}^{n}-p}{S_{k}^{n}}+{ }^{a} \varepsilon_{k}^{n}
$$

where $S_{k}^{n}$ is the current level of the asset price, ${ }^{a} \hat{S}_{k+1}^{n}$ is the reference price level for agent $a,{ }^{a} \varepsilon_{k}^{n}$ is a random variable, independent and identically distributed among agents with zero mean and variance $N \sigma^{2} / n$ where $\sigma$ is a constant. Therefore, we 
assume that the excess demand function consists of two terms: the relative deviation of the proposed price from a reference price level, dependent of the agent, and an exogenous random shock.

We assume that the economy is populated with two types of agents, which differ in their reference price rules. Type 1 agents set the reference price according to the equation:

$$
{ }^{1} \hat{S}_{k+1}^{n}=S_{k}^{n}+S_{k}^{n} \mu_{k}^{n} \frac{1}{n}
$$

where $\mu_{k}^{n}$ is the annualized expected return of the asset price, at time $t_{k}$. Type 1 agents are "rational" since they set the reference price such as the relative price change equals the expected return, $\left({ }^{1} \hat{S}_{k+1}^{n}-S_{k}^{n}\right) / S_{k}^{n}=\mu_{k}^{n} / n$. On the other hand, type 2 agents are "irrational" in that they overreact to every move in asset price that deviates from the expected return. Denoting by $\gamma \in(0,1)$ the overreaction parameter, the expected change for a type 2 agent is assumed to be

$$
\frac{{ }^{2} \hat{S}_{k+1}^{n}-S_{k}^{n}}{S_{k}^{n}}=\mu_{k}^{n} \frac{1}{n}+\gamma\left(\frac{p-S_{k}^{n}}{S_{k}^{n}}-\mu_{k}^{n} \frac{1}{n}\right)
$$

Therefore, type 2 agents take the proposed price $p$ as a signal and employ it in determining the reference price level:

$$
{ }^{2} \hat{S}_{k+1}^{n}=S_{k}^{n}+S_{k}^{n}(1-\gamma) \mu_{k}^{n} \frac{1}{n}+\gamma\left(p-S_{k}^{n}\right)
$$

The market price of the asset at time $t_{k+1}, S_{k+1}^{n}$, follows from the equilibrium condition that the total excess demand is zero, $\sum_{a=1}^{N}{ }^{a} e_{k+1}^{n}\left(S_{k+1}^{n}\right)=0$. Denoting by $\mathcal{X}_{k}^{n} \in(0,1)$ the proportion of type 2 agents at time $t_{k}$, it follows that the asset price 
follows the following stochastic difference equation:

$$
S_{k+1}^{n}-S_{k}^{n}=\mu_{k}^{n} S_{k}^{n} \frac{1}{n}+\frac{\sigma}{1-\gamma \mathcal{X}_{k}^{n}} S_{k}^{n} \frac{\varepsilon_{k+1}^{n}}{\sqrt{n}}
$$

where

$$
\frac{\sigma}{\sqrt{n}} \varepsilon_{k+1}^{n}:=\frac{1}{N} \sum_{a=1}^{N}{ }^{a} \varepsilon_{k+1}^{n},
$$

$E\left(\varepsilon_{k+1}^{n}\right)=0, \operatorname{VAR}\left(\varepsilon_{k+1}^{n}\right)=1$.

Consider for now that the fraction of type 2 agents is constant, $\mathcal{X}_{k}^{n}=\mathcal{X}$. The continuous time limit of equation (4) is given by

$$
\frac{d S_{t}}{S_{t}}=\mu_{t} d t+\sigma \frac{1}{1-\gamma \mathcal{X}} d W_{t}
$$

where $W_{t}$ is a Brownian motion.

Therefore, under the assumption of constant proportion of "irrational" agents, one obtains a constant volatility model. The existence of "irrational" agents induces a multiplication effect of fundamental volatility. More precisely, the asset price volatility is equal to the fundamental volatility $\sigma$ multiplied by $1 /(1-\gamma \mathcal{X})$. Stochastic volatility in this model can result from the existence of a stochastic fundamental volatility and/or from a stochastic multiplication factor. It what follows, we assume that the fundamental volatility is constant and that the fraction of "irrational" agents is stochastic and described by a herding mechanism.

We employ a herding process similar to Alfarano, Lux and Wagner (2008). More specifically, we assume that the fraction of type 2 agents follows a birth-death process 
with the following transition probabilities:

$$
\begin{aligned}
& P\left(\mathcal{X}_{k+1}^{n}=\mathcal{X}+\frac{1}{N} \mid \mathcal{X}_{k}^{n}=\mathcal{X}\right)=N^{2}(1-\mathcal{X})\left(b a \frac{1}{N}+\frac{c^{2}}{2} \mathcal{X}\right) \frac{1}{n}+o\left(\frac{1}{n}\right) \\
& P\left(\mathcal{X}_{k+1}^{n}=\mathcal{X}-\frac{1}{N} \mid \mathcal{X}_{k}^{n}=\mathcal{X}\right)=N^{2} \mathcal{X}\left(b(1-a) \frac{1}{N}+\frac{c^{2}}{2}(1-\mathcal{X})\right) \frac{1}{n}+o\left(\frac{1}{n}\right)
\end{aligned}
$$

where $a \in(0,1), b, c>0$ are constants.

These transition rates have a straightforward interpretation. At a given moment, there are $N(1-\mathcal{X})$ type 1 agents. The rate that each of these agents can turn into a type 2 agent has two components: an individual component $(b a)$ that is independent of the number of type 2 agents and a herding component $\left(\frac{c^{2}}{2} N \mathcal{X}\right)$ depending of the number of type 2 agents that quantifies the effect of mass pressure. Therefore, the transition rate of a "birth" event (the transformation of a type 1 agent into a type 2 agent) is $N(1-\mathcal{X})\left(b a+\frac{c^{2}}{2} N \mathcal{X}\right)$. Similarly, one can argue that the transition rate of a "death" event (the transformation of a type 2 agent into a type 1 agent) is $N \mathcal{X}\left(b(1-a)+\frac{c^{2}}{2} N(1-\mathcal{X})\right)$. Note that we assume an asymmetry in the individual components of the two transition rates. If one imposes the constraint $a=0.5$, one obtains the same herding mechanism as in Alfarano, Lux and Wagner (2008).

Proposition 1. The continuous time limit of the asset price dynamics, under the assumption that $N$ is large, is given by

$$
\left\{\begin{array}{l}
\frac{d S_{t}}{S_{t}}=\mu_{t} d t+\frac{\sigma}{1-\gamma \mathcal{X}_{t}} d W_{t}^{S} \\
d \mathcal{X}_{t}=b\left(a-\mathcal{X}_{t}\right) d t+c \sqrt{\mathcal{X}_{t}\left(1-\mathcal{X}_{t}\right)} d W_{t}^{\mathcal{X}}
\end{array}\right.
$$

where $W_{t}^{S}$ and $W_{t}^{\mathcal{X}}$ are correlated Brownian motions with $d W_{t}^{S} d W_{t}^{\mathcal{X}}=\rho d t$.

Proof. see the Appendix.

It turns out that one obtains in the continuous time limit a non-affine stochastic 
volatility model with the dynamics of the latent variable, $\mathcal{X}_{t}$, described by a Jacobi diffusion process.

\section{Option pricing}

In contrast to a complete market set up such as in the case of the Black and Scholes (1973) model, the existence of a stochastic latent variable in the present model makes the market incomplete. Therefore, the state-price density is not unique and in order to pricing derivatives one has to impose some risk premium assumptions and define the risk neutral measure as the equivalent martingale measure associated to the postulated state-price density. We assume that the relationship between the risk neutral measure $(Q)$ and the real world measure $(P)$ is given by

$$
\begin{aligned}
& d W_{t}^{S, Q}=d W_{t}^{S, P}+\eta_{S} \frac{\sigma}{1-\gamma \mathcal{X}_{t}} d t \quad \text { and } \\
& d W_{t}^{\mathcal{X}, Q}=d W_{t}^{\mathcal{X}, P}+\eta_{\mathcal{X}} \frac{\sqrt{\mathcal{X}_{t}}}{\sqrt{1-\mathcal{X}_{t}}} d t
\end{aligned}
$$

with the parameters $\eta_{S}$ and $\eta_{\mathcal{X}}$ quantifying the risk premiums associated to the two diffusive sources of risk. Consequently, under the risk neutral measure, the dynamics of the underlying is described by:

$$
\left\{\begin{array}{l}
\frac{d S_{t}}{S_{t}}=(r-q) d t+\sigma \frac{1}{1-\gamma \mathcal{X}_{t}} d W_{t}^{S, Q} \\
d \mathcal{X}_{t}=b^{Q}\left(a^{Q}-\mathcal{X}_{t}\right) d t+c \sqrt{\mathcal{X}_{t}\left(1-\mathcal{X}_{t}\right)} d W_{t}^{\mathcal{X}, Q}
\end{array}\right.
$$

with $d W_{t}^{S, Q} d W_{t}^{\mathcal{X}, Q}=\rho d t, b^{Q}=b+\eta_{\mathcal{X}} c, a^{Q}=\frac{b}{b^{Q}} a$ and the risk-free rate $r$ and the dividend yield $q$ are assumed constant for simplicity.

Since the model is non-affine it is impossible to obtain in closed form the characteristic function of the risk neutral distribution of the log returns and to compute 
option prices by the method of Inverse Fourier Transform. However, one can compute the cumulants of this distribution, as shown in the next proposition.

Proposition 2. The cumulants $\kappa_{j}(\tau, \mathcal{X}), j \geq 1$ of the conditional distribution $\ln S_{t+\tau}-\ln S_{t} \mid \mathcal{X}_{t}=\mathcal{X}$ are given by

$$
\begin{aligned}
& \kappa_{1}(\tau, \mathcal{X})=\left((r-q)-\frac{1}{2} \sigma^{2}\right) \tau+\tilde{\kappa}_{1}(\tau, \mathcal{X}) \\
& \kappa_{2}(\tau, \mathcal{X})=\sigma^{2} \tau+\tilde{\kappa}_{2}(\tau, \mathcal{X}) \\
& \kappa_{j}(\tau, \mathcal{X})=\tilde{\kappa}_{j}(\tau, \mathcal{X}), j \geq 3,
\end{aligned}
$$

where $\tilde{\kappa}_{1}(\tau, \mathcal{X})=f_{1}(\tau, \mathcal{X})$,

$$
\tilde{\kappa}_{j}(\tau, \mathcal{X})=f_{j}(\tau, \mathcal{X})-\sum_{l=1}^{j-1}\left(\begin{array}{l}
j-1 \\
l-1
\end{array}\right) \tilde{\kappa}_{l}(\tau, \mathcal{X}) f_{j-l}(\tau, \mathcal{X}), j \geq 2
$$

and the functions

$$
f_{j}(\tau, \mathcal{X})=\sum_{m=0}^{\infty} f_{j, m}(\tau) P_{m}(\mathcal{X})
$$

with $f_{0,0}(\tau)=1, f_{0, m}(\tau)=0, m>0, P_{m}(\cdot)$ is the $m$-th Jacobi polynomial on the interval $(0,1)$. The functions $f_{j, m}(\tau)$ solve the following system of ordinary differential equations $(O D E)$ :

$$
\dot{f}_{j, m}=-\lambda_{m} f_{j, m}+j \sum_{p=0}^{\infty} g_{m p} f_{j-1, p}+j(j-1) \sum_{p=0}^{\infty} h_{m p} f_{j-2, p}
$$


with $\lambda_{m}=b^{Q} m+\frac{c^{2}}{2} m(m-1), g_{m p}$ and $h_{m p}$ are given by

$$
\begin{aligned}
g_{m p} & =-\frac{\sigma^{2}}{2} \int_{0}^{1}\left(\frac{1}{(1-\gamma x)^{2}}-1\right) P_{m}(x) P_{p}(x) n\left(x ; a^{Q}, b^{Q}, c\right) d x \\
& +\rho \sigma c \int_{0}^{1} \frac{\sqrt{x(1-x)}}{1-\gamma x} P_{m}(x) P_{p}^{\prime}(x) n\left(x ; a^{Q}, b^{Q}, c\right) d x \\
h_{m p} & =\frac{\sigma^{2}}{2} \int_{0}^{1}\left(\frac{1}{(1-\gamma x)^{2}}-1\right) P_{m}(x) P_{p}(x) n\left(x ; a^{Q}, b^{Q}, c\right) d x
\end{aligned}
$$

and $n\left(x ; a^{Q}, b^{Q}, c\right)$ is the probability distribution function of a Beta distribution with parameters $\alpha=\frac{2 b^{Q}}{c^{2}} a^{Q}$ and $\beta=\frac{2 b^{Q}}{c^{2}}\left(1-a^{Q}\right)$.

Proof. see the Appendix.

In order to implement the results in the previous proposition one has to truncate the expansion of $f_{j}(\tau, \mathcal{X})$ in equation $(10)$ after a finite number of terms. From our various experiments it turns out that using the first 40 terms is, in general, enough to ensure the convergence of the expansion of the function on Jacobi polynomials. After deciding the truncation threshold, one can easily compute recursively the functions $f_{j, m}(\tau)$ since the (truncated) system of ODEs in equation (11) has an explicit solution.

Let us suppose we want to determine the theoretical price at time $t$ of a European call option with maturity $t+\tau$ using the model in this paper. In the beginning, we compute the first 40 cumulants of the risk-neutral conditional distribution $\ln S_{t+\tau}-\ln S_{t} \mid \mathcal{X}_{t}=\mathcal{X}$ using Proposition 1. Next, we approximate the probability distribution function using the type C Gram-Charlier series expansion (C-GCSE) truncated after 20 terms by computing the expansion coefficients of the C-GCSE from the first 40 moments/cumulants using the method in Rompolis and Tzavalis (2007). In contrast with the classical type A Gram-Charlier expansion that is employed in the literature (e.g. Jondeau and Rockinger, 2001), the type C expansion guarantees that the values of the risk neutral density will be always positive. On the downside, 
there is no closed form formula for option prices when approximating the risk neutral probability distribution function with a C-GCSE.

Finally, we employ the general closed form option pricing formula obtained in Necula, Drimus and Farkas (2013). This method for option pricing is based on the fact that a probability density can be represented as a Gauss-Hermite series expansion (GHSE). More specifically, let us denote by $p_{L}(x ; \tau, s, \mathcal{X})$ the C-GCSE approximation of the probability distribution function of $\ln S_{t+\tau}-\ln S_{t} \mid \mathcal{X}_{t}=\mathcal{X}$ obtained by employing the first $L$ cumulants. Then, the GHSE approximation of $p_{L}(x ; \tau, s, \mathcal{X})$ is given by:

$$
p_{L}(x ; \tau, \mathcal{X})=\frac{1}{\sqrt{\kappa_{2}(\tau, \mathcal{X})}} z\left(\frac{x-\kappa_{1}(\tau, \mathcal{X})}{\sqrt{\kappa_{2}(\tau, \mathcal{X})}}\right) \sum_{n=0}^{\infty} a_{n}(\tau, \mathcal{X}) H_{n}\left(\frac{x-\kappa_{1}(\tau, \mathcal{X})}{\sqrt{\kappa_{2}(\tau, \mathcal{X})}}\right)
$$

where $H_{n}(x)$ denotes the $n$ th-order "physicists" Hermite polynomial and $z(x)$ is the standard Gaussian density. Using the orthogonality condition of the "physicists" Hermite polynomials, it follows that the Gauss-Hermite expansion coefficients can be computed as:

$$
a_{n}(\tau, \mathcal{X})=\frac{\sqrt{\pi}}{2^{n-1} n !} \int_{-\infty}^{\infty} z\left(\frac{x-\kappa_{1}(\tau, \mathcal{X})}{\sqrt{\kappa_{2}(\tau, \mathcal{X})}}\right) H_{n}\left(\frac{x-\kappa_{1}(\tau, \mathcal{X})}{\sqrt{\kappa_{2}(\tau, \mathcal{X})}}\right) p_{L}(x ; \tau, \mathcal{X}) d x
$$

The Gauss-Hermite series expansion is an attractive alternative for approximating the risk-neutral density due to its improved convergence for heavy tailed distributions and because it allows for a closed form formula for pricing European options as shown in Necula, Drimus and Farkas (2013). More specifically, the price of at time $t$ of a European call option with strike price $K$ and maturity $t+\tau$ is given by:

$$
c\left(S_{t}, K, \tau, \mathcal{X}_{t}\right)=S_{t} e^{-q \tau} \Pi_{1}\left(\tau, S_{t}, \mathcal{X}_{t}\right)-K e^{-r \tau} \Pi_{2}\left(\tau, S_{t}, \mathcal{X}_{t}\right)
$$


with

$$
\begin{aligned}
& \Pi_{1}\left(\tau, S_{t}, \mathcal{X}_{t}\right)=\exp \left(\kappa_{1}(\tau, \mathcal{X})-(r-q) \tau+\frac{\kappa_{2}(\tau, \mathcal{X})}{2}\right) \sum_{n=0}^{\infty} a_{n}\left(\tau, \mathcal{X}_{t}\right) I_{n}\left(\tau, S_{t}, \mathcal{X}_{t}\right) \text { and } \\
& \Pi_{2}\left(\tau, S_{t}, \mathcal{X}_{t}\right)=\sum_{n=0}^{\infty} a_{n}\left(\tau, \mathcal{X}_{t}\right) J_{n}\left(\tau, S_{t}, \mathcal{X}_{t}\right)
\end{aligned}
$$

where $I_{n}\left(\tau, S_{t}, \mathcal{X}_{t}\right)$ and $J_{n}\left(\tau, S_{t}, \mathcal{X}_{t}\right)$ satisfy simple recursion equations that are described in Necula, Drimus and Farkas (2013).

When truncating the GHSE one has to make sure that the obtained approximation is a proper risk-neutral density, such that it is positive, has unit mass and the martingale restriction is observed. Since the truncation is done after a large number of terms (20 or 30) these restrictions are naturally obtained given the good convergence properties of the GHSE with the expansion coefficients computed from 13. However, to make sure the restrictions are valid, a second step was applied in the empirical study and consisted in obtaining the GH expansion coefficients by minimizing the sum the squared differences between the values, in an appropriate range, of the GHSE approximation and of the "real" density computed using C-GCSE and imposing the required constraints. This optimization is quite efficient since the GHSE approximation and the constrains are linear in the expansion coefficients.

The performance of the Herding model is assessed empirically by comparison to benchmark models. We apply the well known and frequently used affine onefactor Heston model as well as the two-factor double Heston model proposed by Christoffersen, Heston and Jacobs (2009). For details the reader is referred to the appendix. 


\section{Data and estimation methodology}

For the empirical performance analysis we employ the historical series of the daily close price of S\&P 500 OTM index call options for the period January 1996 - December 2013. We use S\&P 500 index options because they have the highest trading volume of all European style options, because they are frequent applied in similar studies and because of the subsequent availability of data. We derive the respective empirical ITM prices by the put-call parity. The daily quoted mid-prices are sourced from the Option Metrics IVY Database.

Since the optimization of the proposed model is computationally intensive we apply the analysis on each Wednesday and consecutive Thursday for each week between January 1996 and December 2013. The database likewise allows to source the annualized dividend yield and the zero-coupon interest rate curve, matching the date and maturity of the respective options.

Short term options exhibit a stronger volatility smile and consequently it is more challenging to fit a proposed model to their prices (following for example Bakshi et al. (1997)). Accordingly we focus our empirical study on short term options defined as options with a maturity of equal or less than 3 months, measured in trading days. On the low end of the maturity we take only into account options with a maturity of at least one week to prevent liquidity related biases (again following Bakshi et al. (1997)). Other commonly applied exclusion filters are used (for example Bakshi et al. (1997) and Bardgett et al. (2013)): We only include options which exhibit a strictly positive bid price, a positive and finite moneyness and fulfill the no-arbitrage condition:

$$
\text { Call }>\max \left(0, S e^{-q \tau}-K e^{-r f \tau}\right), P u t>\max \left(0, K e^{-r f \tau}-S e^{-q \tau}\right)
$$

Following a description of the short term option sample: The summary of the average 
Table 1: Average implied volatility and volume of sample

\begin{tabular}{|c|c|c|c|c|c|c|c|}
\hline & \multicolumn{7}{|c|}{ Months-to-Expiration } \\
\hline & $\begin{array}{r}\text { Between } 1 \\
\text { Week and } \\
1 \text { Month }\end{array}$ & & $\begin{array}{r}\text { Between } 1 \\
\text { and } 2 \\
\text { Months }\end{array}$ & & $\begin{array}{r}\text { Between } 2 \\
\text { and } 3 \\
\text { Months }\end{array}$ & & \\
\hline Moneyness & $\begin{array}{l}\text { Average of } \\
\text { Market IV }\end{array}$ & $\begin{array}{r}\text { Number of } \\
\text { Options }\end{array}$ & $\begin{array}{l}\text { Average of } \\
\text { Market IV }\end{array}$ & $\begin{array}{r}\text { Number of } \\
\text { Options }\end{array}$ & $\begin{array}{l}\text { Average of } \\
\text { Market IV }\end{array}$ & $\begin{array}{r}\text { Number of } \\
\text { Options }\end{array}$ & $\begin{array}{r}\text { Total } \\
\text { Number of } \\
\text { Options }\end{array}$ \\
\hline$<0.94$ & 0.3141 & 12552 & 0.2972 & 37619 & 0.2869 & 27968 & 78139 \\
\hline $0.94 \leq,<0.97$ & 0.1797 & 6671 & 0.1797 & 11476 & 0.1902 & 6407 & 24554 \\
\hline $0.97 \leq, 1$ & 0.1656 & 7540 & 0.175 & 12477 & 0.1869 & 7144 & 27161 \\
\hline $1 \leq,<1.03$ & 0.1651 & 7329 & 0.1751 & 12388 & 0.1864 & 7105 & 26822 \\
\hline $1.03 \leq,<1.06$ & 0.1705 & 6679 & 0.1764 & 11068 & 0.1881 & 6230 & 23977 \\
\hline$\geq 1.06$ & 0.2924 & 15199 & 0.2845 & 42342 & 0.2788 & 30604 & 88145 \\
\hline $\begin{array}{l}\text { Total Number } \\
\text { of Options }\end{array}$ & & 55970 & & 127370 & & 85458 & 268798 \\
\hline
\end{tabular}

The table shows the average Black Scholes implied volatility per maturity band and moneyness band including the number of options in the sample. The moneyness is the ratio of the spot and strike or the strike and spot depending on ITM/OTM and Put/Call characteristic of the respective option. The maturity is measured in trading days.

implied volatilities shows the expected string volatility smiles. The total number of 268,798 short term options is including the derived ITM options and after the application of all exclusion filters taking into account that only those options remained in the sample with a positive price for all applied models simultaneously.

To fit each model to the empirical options we employ a combination of differential evolution (DE) and non-linear least squares optimization. Searching the best input parameters we have come to the conclusion that a combination of the two algorithms performs often significantly better than if only one is chosen. The applied DE is implemented in the swarmOps package for Matlab available online*. The DE implementation in the swarmOps package is the basic variant developed by Storn and Price (1997) applying darwinian evolution techniques such as inheritance, mutation, recombination, crossover and selection.

The DE is a direct stochastic search algorithm, aiming to find the global optimum and capable of running in parallel. It essentially initiates a predefined amount of ran-

*http://www.hvass-labs.org/projects/swarmops/matlab/ 
dom parameter vectors which are updated until convergence to the global minimum. In more detail, a sample of predefined size, typically more than three, of random parameter vectors is built, covering the parameter space. These candidate solutions are often uniformly distributed. For each of these candidate vectors the following steps are performed: First, three vectors are randomly chosen from amongst the candidate vectors. From these, the weighted difference of two vectors is added to the third vector to yield a mutated vector. The third vector is called target vector. The weight of the difference or rather the amplitude is chosen by the user from $[0,2]$. Then the mutated vector and the respective target vector are crossed over to result in the trial vector. The method of crossover is basically choosing for each value in the trial vector either the value of the mutated vector or the target vector depending on the level of random uniform number and a predefined threshold Storn and Price (1997) . The crossover assures that the new trial vector differs from the initial target vector in a minimum amount of components. If the trial vector yields a lower cost than the target vector the later is replaced by the trial vector. This step is the selection step and the cost function can be a fitness or objective function. After going through all candidate vectors the remaining target- and trial vectors build the new sample for the next generation of updates.

One of the main difference to traditional Evolution algorithms is that the randomly chosen population vectors perturb an existing vector within the population instead of applying a chosen probability distribution function for the evolution of vectors Storn and Price (1997).

We use the resulting global optimum parameters as input in the more classic nonlinear least squares optimization algorithm, implemented in Matlab. In consideration of the complexity of the herding model function we apply the multistart version of the trust region reflective algorithm. 


\section{Empirical results}

The combination of optimization algorithms is applied to minimize the fitness of implied volatilities: the square root of the average of the difference between the Black Scholes implied volatility on the observed option premium and the Black Scholes implied volatility on the model premium per trading day. The objective function is thus:

$$
\text { fitness }_{t}:=\min _{t} \sqrt{\frac{\sum_{i=1}^{n_{t}}\left(I V_{i}-\widehat{I V}_{i}\left(a_{t}, b_{t}, c_{t}, \gamma_{t}, \sigma_{t}, \rho_{t}, \chi_{t}\right)\right)}{n_{t}}}
$$

$n_{t}$ being the number of options on day t to which the structural parameters are fitted and $I V, \widehat{I V}$ are the Black Scholes implied volatilities for the observed and modelled option price respectively.

The minimization of the Black Scholes implied volatilities is more comparable between options of different strike prices and maturities (e.g. Andersen, Fusari and Todorov, 2015). By this procedure one finds a set of structural parameters for all short term options on a chosen day. We likewise apply the parameters estimated on data of each Wednesday from 1996 - 2013 on the market data of following Thursday to compute out of sample estimates. These procedure is repeated equally for the benchmark models.

Table 2 reports the descriptive statistics of the daily parameters estimated using the above methodology for the one-factor models employed in the analysis.

Table 2: Average of estimated daily parameters for short term options

\begin{tabular}{cccccccccccc}
\hline & & $a_{Q}$ & $b_{Q}$ & $\mathrm{c}$ & $\gamma$ & $\chi$ & $\sigma$ & $\rho$ & $\theta$ & $\kappa$ & $\mathrm{v}$ \\
\hline \multirow{2}{*}{ Herding Model } & Average & 0.4 & 2.71 & 1.28 & 0.95 & 0.64 & 0.07 & -0.8 & & & \\
& Stdev & 0.19 & 0.7 & 0.3 & 0.02 & 0.14 & 0.04 & 0.11 & & & \\
& Skew & -0.47 & 0.15 & 1.22 & -1.16 & -0.39 & 1.26 & 1.02 & & & \\
Heston Model & Average & & & & & & 0.05 & -0.89 & 0.94 & 4.96 & 0.54 \\
& Stdev & & & & & & 0.03 & 0.13 & 3.9 & 4.91 & 0.25 \\
& Skew & & & & & & 3.39 & 1.51 & 6.41 & 1.8 & 0.47 \\
\hline
\end{tabular}

Average, standard deviation and skew of the parameters for the Herding and Heston model on the whole sample of daily sort term options from 1996-2013.

Overall the parameters are consistent for the one-factor models within the analysis 
on the short term options. The latent volatilities, v, range around the expected levels and are similar between the models.

To assess the relative model misspecification we compare the Black-Scholes volatilities implied by modelled prices of the Herding model, its benchmarks and the market implied volatility. Since the volatility smile is strongest for options with a maturity of less than 60 days we focus on short term options with the respective maturity. The following graph shows the average implied volatilities over the whole sample. On

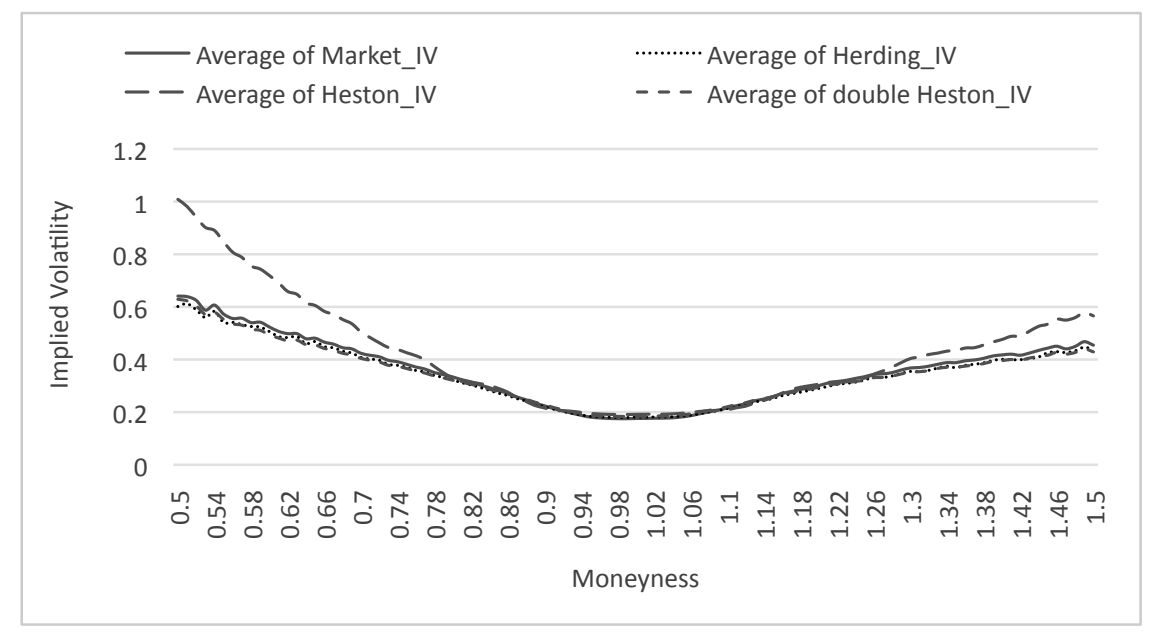

Figure 1: Comparison between Model- and Market implied Volatilities on the Full Range of Moneyness

the whole range of moneyness, above and below 1.5 and 0.5 respectively the Heston model implied volatilities tend to be higher then the Herding model implied, the double Heston model and the market implied volatility.

The Herding implied volatility is on average closer to the market implied volatility. In between a moneyness of 0.9 and 1.1 the fit of all three models seems agreeable, as seen in figure 2. Focusing on the most recent year, 2013, within a moneyness from 0.9 to 1.1, the order is similar: the Heston model implied volatilities are in tendency higher than the other volatilities. On the other hand the double Heston models volatility is closer to the market in this specific year as can be seen in figure 


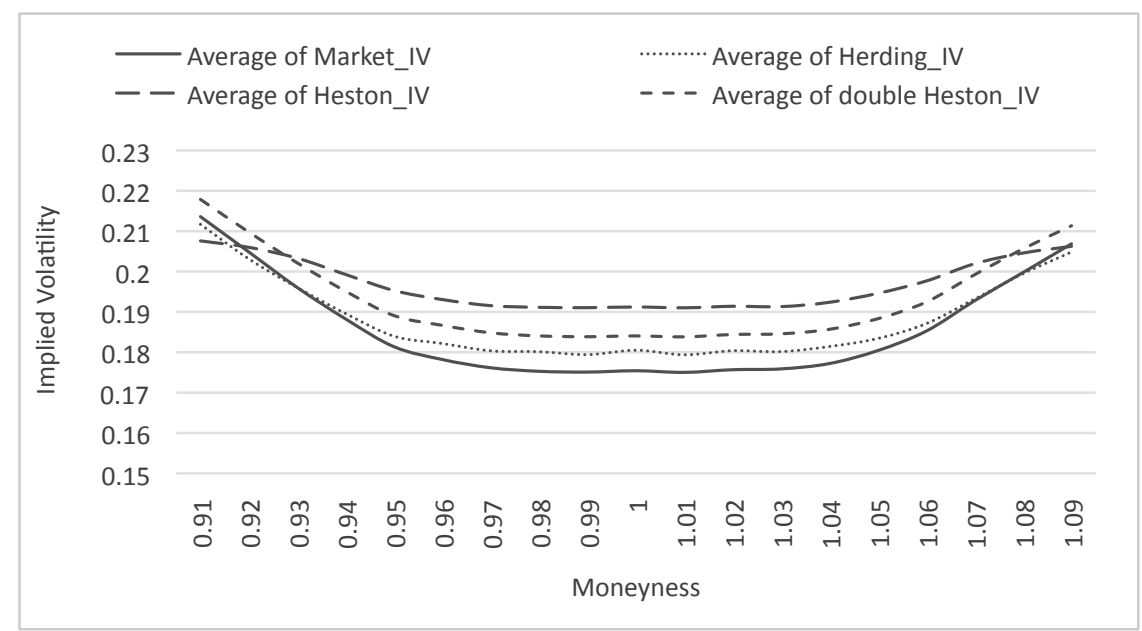

Figure 2: Comparison between Model- and Market implied Volatilities between a Moneyness of $0.9-1.1$

3. Overall all models depict the expected $U$ shape of the implied volatilities and we

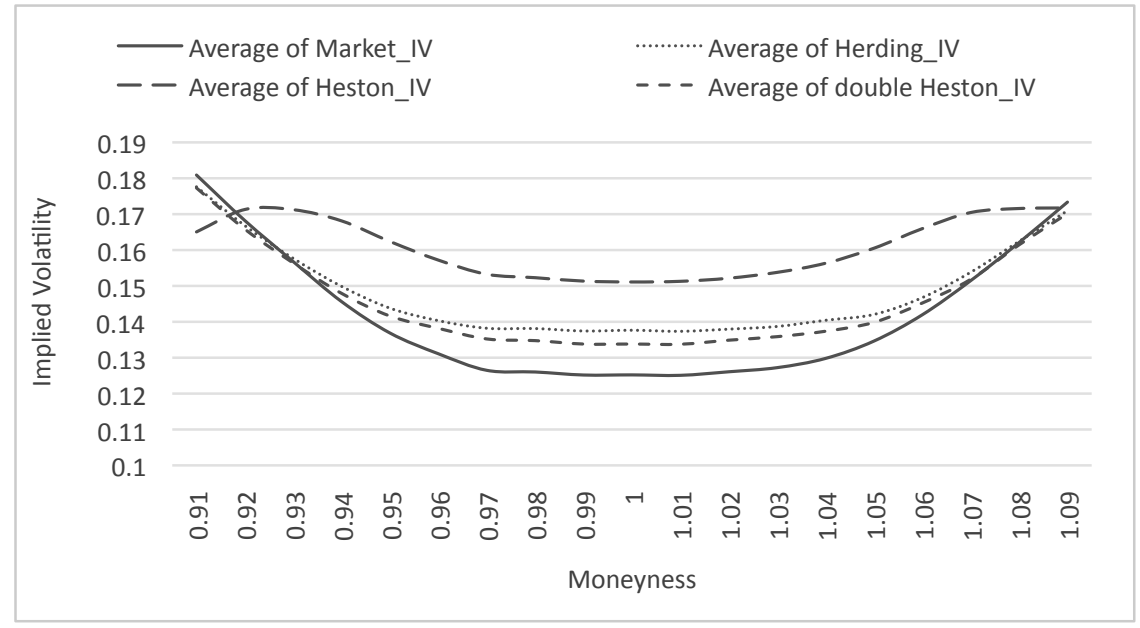

Figure 3: Comparison between Model- and Market implied Volatilities between a Moneyness of 0.9-1.1, in 2013 only.

cannot find any indication for a model misspecification.

The in- and out of sample performance analysis are each done for all three (one and two factor) models and the average fitness per year is reported.

The average in-sample fitness the Herding model in comparison to the Heston model is lower in $83 \%$ of the cases but with respect the double Heston model in only 
one year.

Table 3: Comparison of fitness by Heston model and double Heston model vs Herding model with In-Sample Data

\begin{tabular}{cccc}
\hline Year & $\begin{array}{c}\text { Average of } \\
\text { Heston Squared } \\
\text { Error }\end{array}$ & $\begin{array}{c}\text { Average of } \\
\text { double Heston } \\
\text { Squared Error }\end{array}$ & $\begin{array}{c}\text { Average of } \\
\text { Herding } \\
\text { Squared Error }\end{array}$ \\
\hline 1996 & 0.025 & 0.011 & 0.043 \\
1997 & 0.033 & 0.021 & 0.025 \\
1998 & 0.052 & 0.029 & 0.027 \\
1999 & 0.047 & 0.021 & 0.05 \\
2000 & 0.041 & 0.021 & 0.038 \\
2001 & 0.031 & 0.02 & 0.03 \\
2002 & 0.036 & 0.017 & 0.041 \\
2003 & 0.041 & 0.012 & 0.036 \\
2004 & 0.04 & 0.013 & 0.029 \\
2005 & 0.044 & 0.016 & 0.027 \\
2006 & 0.043 & 0.016 & 0.04 \\
2007 & 0.041 & 0.015 & 0.026 \\
2008 & 0.042 & 0.015 & 0.033 \\
2009 & 0.082 & 0.028 & 0.036 \\
2010 & 0.064 & 0.021 & 0.031 \\
2011 & 0.078 & 0.023 & 0.027 \\
2012 & 0.055 & 0.016 & 0.032 \\
2013 & 0.05 & 0.013 & 0.023 \\
\hline
\end{tabular}

Average fitness of the short term options in the sample per year for all models. The fitness is calculated in-sample.

Fitting the models to daily short term option prices the Herding model is around 1.5 times as accurate as the Heston model and around half as accurate as the double Heston model.

Nonetheless, the outperformance by the Herding model of the Heston model could be related to the fact that the Herding model has more parameters. We thus enhance our assessment by an out-of-sample analysis. Using different data than the dataset the model was fitted to, reduces the risk of the results being due to the amount of parameters as overfitting is likely to occur if the former was the case.

Like the in-sample comparison the Herding model outperforms the Heston model and is less accurate than the double Heston model. In general the difference between the Herding and the benchmark models is reduced when applied to out of sample data. Fitting to short term options the Herding model is around 1.3 times as accurate as the 
Table 4: Comparison of fitness by Heston model and double Heston model vs Herding model, with Out-of-Sample Data

\begin{tabular}{cccc}
\hline Year & $\begin{array}{c}\text { Heston - Short } \\
\text { Term Oos }\end{array}$ & $\begin{array}{c}\text { double Heston } \\
\text { Short Term Oos }\end{array}$ & $\begin{array}{c}\text { Herding Short } \\
\text { Term Oos }\end{array}$ \\
\hline 1996 & 0.03 & 0.025 & 0.049 \\
1997 & 0.035 & 0.035 & 0.032 \\
1998 & 0.057 & 0.039 & 0.032 \\
1999 & 0.052 & 0.033 & 0.054 \\
2000 & 0.048 & 0.035 & 0.044 \\
2001 & 0.037 & 0.035 & 0.034 \\
2002 & 0.042 & 0.034 & 0.046 \\
2003 & 0.042 & 0.024 & 0.037 \\
2004 & 0.043 & 0.023 & 0.035 \\
2005 & 0.046 & 0.026 & 0.028 \\
2006 & 0.046 & 0.035 & 0.033 \\
2007 & 0.043 & 0.026 & 0.033 \\
2008 & 0.04 & 0.031 & 0.035 \\
2009 & 0.082 & 0.037 & 0.032 \\
2010 & 0.067 & 0.033 & 0.036 \\
2011 & 0.085 & 0.034 & 0.04 \\
2012 & 0.056 & 0.02 & 0.039 \\
2013 & 0.05 & 0.016 & 0.025 \\
\hline
\end{tabular}

Average fitness of the short term options per year for all models. The fitness is calculated out-of-sample.

Heston and 0.8 times as accurate as the double Heston model. The Herding model is again more accurate than the Heston model in $83 \%$ of the cases and outperforms the double Heston model in $28 \%$ of the tested years.

Focusing on analyzing best performing models on days instead of years in the out-of-sample analysis of short term options, the Herding model provides the most accurate prices in 45\%, the double Heston model in 50\% and the Heston model in 5\% of the cases. The following table 5 shows in detail the share of best performing daily out-of-sample analysis for each year in the sample.

In eight out of the 18 years in the sample, the Herding model returns the most accurate estimates for the majority of the days in those years. The double Heston model is likewise the most accurate model for the majority of days during another eight years. The Heston model is never outperforming the other two models from the point of view of offering better estimates in the majority of days in a given year. The 
Table 5: Daily Share of Best Performing ModelOut-of-sample.

\begin{tabular}{cccc}
\hline Year & Herding & Heston & $\begin{array}{c}\text { double } \\
\text { Heston }\end{array}$ \\
\hline 1996 & 0.45 & 0.1 & 0.45 \\
1997 & 0.43 & 0.05 & 0.53 \\
1998 & 0.71 & 0.1 & 0.2 \\
1999 & 0.65 & 0.08 & 0.27 \\
2000 & 0.62 & 0.04 & 0.34 \\
2001 & 0.53 & 0.14 & 0.33 \\
2002 & 0.44 & 0.14 & 0.42 \\
2003 & 0.24 & 0.04 & 0.72 \\
2004 & 0.3 & 0.02 & 0.68 \\
2005 & 0.46 & 0 & 0.54 \\
2006 & 0.54 & 0.1 & 0.35 \\
2007 & 0.44 & 0.03 & 0.53 \\
2008 & 0.53 & 0.05 & 0.42 \\
2009 & 0.56 & 0 & 0.44 \\
2010 & 0.49 & 0.02 & 0.49 \\
2011 & 0.37 & 0 & 0.63 \\
2012 & 0.31 & 0 & 0.69 \\
2013 & 0.18 & 0 & 0.82 \\
\hline
\end{tabular}

The table shows for each model the percentage of days per analysed year where the respective model performed best. The analysis is out-ofsample.

results indicate that the Herding model can compete with the double Heston model since both are most accurate in the same number of cases. On the other hand, the double Heston dominates the Herding model in the case the annual average fitness is compared.

With regard to the possibility that the results are driven by a potentially changing underlying sample structure we are investigating key characteristics of the underlying short term options, shown in table 6 as daily averages per year.

The number of options analysed per day as well as the characteristics of the options in the sample do not exhibit any systematic deviation or trend, potentially biasing the derived results. We thus conclude that the results are entirely due to the characteristics of the models. 
Table 6: Characteristics of Short Term Options per Year

\begin{tabular}{lcccccc}
\hline $\begin{array}{l}\text { Row } \\
\text { Labels }\end{array}$ & $\begin{array}{c}\text { Average of } \\
\text { Spot }\end{array}$ & $\begin{array}{c}\text { Average of } \\
\text { Implied } \\
\text { Volatility }\end{array}$ & $\begin{array}{c}\text { Average of } \\
\text { Share of Put } \\
\text { Options }\end{array}$ & $\begin{array}{c}\text { Average of } \\
\text { Maturity }\end{array}$ & $\begin{array}{c}\text { Average of } \\
\text { Strike }\end{array}$ & $\begin{array}{c}\text { Daily Average } \\
\text { Number of } \\
\text { Options }\end{array}$ \\
\hline 1996 & 672.74 & 0.18 & 0.64 & 0.14 & 647.42 & 126 \\
1997 & 876.33 & 0.24 & 0.66 & 0.14 & 832.26 & 162 \\
1998 & 1090.43 & 0.28 & 0.68 & 0.13 & 1018.81 & 162 \\
1999 & 1328.34 & 0.27 & 0.63 & 0.13 & 1250.58 & 159 \\
2000 & 1422.46 & 0.24 & 0.53 & 0.14 & 1378.61 & 145 \\
2001 & 1195.67 & 0.27 & 0.55 & 0.14 & 1164.23 & 140 \\
2002 & 988.77 & 0.28 & 0.53 & 0.14 & 961.26 & 136 \\
2003 & 968.35 & 0.24 & 0.62 & 0.13 & 920.02 & 136 \\
2004 & 1131.15 & 0.18 & 0.64 & 0.13 & 1071.18 & 148 \\
2005 & 1207.24 & 0.15 & 0.6 & 0.13 & 1162.11 & 165 \\
2006 & 1316.72 & 0.15 & 0.63 & 0.14 & 1266.74 & 214 \\
2007 & 1480.75 & 0.2 & 0.65 & 0.13 & 1407.21 & 307 \\
2008 & 1289.6 & 0.26 & 0.54 & 0.14 & 1259.55 & 344 \\
2009 & 956.98 & 0.35 & 0.65 & 0.14 & 870.72 & 508 \\
2010 & 1140.27 & 0.28 & 0.69 & 0.14 & 1031.97 & 506 \\
2011 & 1259.07 & 0.3 & 0.7 & 0.14 & 1132.01 & 616 \\
2012 & 1381.4 & 0.23 & 0.69 & 0.13 & 1269.14 & 724 \\
2013 & 1595.53 & 0.18 & 0.7 & 0.13 & 1490.41 & 850 \\
\hline
\end{tabular}

The table summarizes specific characteristics of short term options per year.

\section{Concluding remarks}

We developed an endogenous, non-affine stochastic volatility model where the volatility induced by fundamentals is amplified due to the interaction and herding of the agents involved in trading a financial asset. For pricing options in this model we employed the methodology based on Gauss-Hermite series expansion developed in Necula, Drimus and Farkas (2013). The option pricing performance of the new nonaffine one-factor model was tested in comparison to the classical one-factor model proposed by Heston and the affine two-factor double Heston model. We employed daily data on S\&P 500 short term options for the period January 1996 - August 2013. The parameters of all models were estimated by the same methodology consisting on a combination of differential evolution and non-linear least squares optimization of the implied volatility mean squared error. The empirical analysis points out that the herding model is more accurate than the affine one-factor model both in- as well as 
out of sample and is comparable with the affine two-factor model in half of the cases under scrutiny.

\section{References}

Alfarano, S., T. Lux, and F. Wagner (2008). Time-variation of higher moments in a financial market with heterogeneous agents: An analytical approach. Journal of Economic Dynamics and Control, 32(1), 101-136.

Andersen, T., N. Fusari, and V. Todorov (2015). The risk premia embedded in index options. Journal of Financial Economics, 117, 558-584.

Bakshi, G., C. Cao, and Z. Chen (1997). Empirical performance of alternative option pricing models. Journal of Finance, 52, 2003-2049.

Bardgett, C., E. Gourier, and M. Leippold (2013). Inferring volatility dynamics and risk premia from the S\&P500 and VIX markets. Working Paper, University of Zurich.

Bates, D. S. (1996). Jumps and stochastic volatility: exchange rate processes implicit in Deutsche Mark options. Review of Financial Studies, 9, 69-107.

Black, F. (1976). Studies of stock price volatility changes. In Proceedings of the 1976 Meetings of the Business and Economic Statistics Section, American Statistical Association. 177-181.

Black, F. and M. Scholes (1973). The pricing of options and corporate liabilities. Journal of Political Economy, 81, 637-659.

Broadie, M., M. Chernov, and M. Johannes (2007). Model specification and risk premia: evidence from futures options. Journal of Finance, 62, 1453-1490. 
Christoffersen, P., S. Heston, and K. Jacobs (2009). The shape and term structure of the index option smirk: Why multifactor stochastic volatility models work so well. Management Science, 55, 1914-1932.

Drimus, G. G. (2012). Options on realized variance by transform methods: A nonaffine stochastic volatility model. Quantitative Finance, 12, 1679-1694.

Duan, J. and C. Yeh (2010). Jump and volatility risk premiums implied by VIX. Journal of Economic Dynamics and Control, 34, 2232-2244.

Eraker, B. (2004). Do stock prices and volatility jump? Reconciling evidence from spot and option prices. Journal of Finance, 59, 1367-1404.

Follmer, H., U. Horst, and A. Kirman (2005). Equilibria in financial markets with heterogeneous agents: a probabilistic perspective. Journal of Mathematical Economics, 41, 123-155.

Follmer, H. and M. Schweizer (1993). A microeconomic approach to diffusion models for stock prices. Mathematical Finance, 3, 1-23.

Gouriéroux, G., E. Renault, and P. Valery (2007). Diffusion processes with polynomial eigenfunctions. Annales d'économie et de statistique, 85, 115-130.

Heston, S. L. (1993). A closed-form solution for options with stochastic volatility with applications to bond and currency options. Review of Financial Studies, 6, $327-343$.

Horst, U. (2005). Financial price fluctuation in a stock market model with many interacting agents. Economic Theory, 25, 917-932.

Jondeau, E. and M. Rockinger (2001). Gram-Charlier densities. Journal of Economic Dynamics and Control, 25, 1457-1483. 
Jones, C. (2003). The dynamics of the stochastic volatility: Evidence from underlying and options markets. Journal of Econometrics, 116, 181-224.

Kaeck, A. and C. Alexander (2012). Volatility dynamics for the S\&P 500: Further evidence from non-affine, multi-factor jump diffusions. Journal of Banking $\& 5$ Finance, $36,3110-3121$.

Moodley, N. (2005). The heston model: A practical approach. Available online in internet: http://math.nyu.edu/ atm262/fall06/compmethods/a1/ nimalinmoodley ·pdf.

Necula, C., G. Drimus, and W. Farkas (2013). A general closed form option pricing formula. http://papers.ssrn.com/sol3/papers.cfm?abstract_id=2210359.

Pan, J. (2002). The jump-risk premia implicit in options: Evidence from an integrated time series study. Journal of Financial Economics, 63, 3-50.

Polyanin, A. D. (2001). Handbook of Linear Partial Differential Equations for Engineers and Scientists. Chapman and Hall.

Rompolis, L. S. and E. Tzavalis (2007). Retrieving risk neutral densities based on risk neutral moments through a Gram-Charlier series expansion. Mathematical and Computer Modelling, 46, 225-234.

Storn, R. and K. Price (1997). Differential evolution a simple and efficient heuristic for global optimization over continuous spaces. Journal of Global Optimization, 11, 341-359. 


\section{Appendix}

\section{Proofs}

Proof of Proposition 1. From (4) it follows that the equilibrium price at time $t_{k+1}$ is

$$
S_{k+1}^{n}=S_{0}^{n}+\sum_{i=0}^{k} \mu_{i}^{n} S_{i}^{n} \frac{1}{n}+\sum_{i=0}^{k} \frac{\sigma}{1-\gamma \mathcal{X}_{i}^{n}} S_{i}^{n} \frac{\varepsilon_{i+1}^{n}}{\sqrt{n}}
$$

Using the transition probabilities in equation (6) we have that:

$$
\begin{aligned}
E\left(\mathcal{X}_{k+1}^{n}-\mathcal{X}_{k}^{n} \mid \mathcal{X}_{k}^{n}=\mathcal{X}\right) & =b(a-\mathcal{X}) \frac{1}{n}+o\left(\frac{1}{n}\right) \\
E\left(\left(\mathcal{X}_{k+1}^{n}-\mathcal{X}_{k}^{n}\right)^{2} \mid \mathcal{X}_{k}^{n}=\mathcal{X}\right) & =\left(c^{2} \mathcal{X}(1-\mathcal{X})+\frac{b(a(1-\mathcal{X})+\mathcal{X}(1-a))}{N}\right) \frac{1}{n} \\
& +o\left(\frac{1}{n}\right) \text { and } \\
E\left(\left(\mathcal{X}_{k+1}^{n}-\mathcal{X}_{k}^{n}\right)^{j} \mid \mathcal{X}_{k}^{n}=\mathcal{X}\right) & =o\left(\frac{1}{n}\right), j \geq 3 .
\end{aligned}
$$

For $N$ large, the term $\frac{b(a(1-\mathcal{X})+\mathcal{X}(1-a))}{N}$ in equation (A.3) is negligible compared to $c^{2} \mathcal{X}(1-\mathcal{X})$ and it follows that

$$
\mathcal{X}_{k+1}^{n}=\mathcal{X}_{0}^{n}+\sum_{i=0}^{k}\left(\mathcal{X}_{i+1}^{n}-\mathcal{X}_{i}^{n}\right)=\mathcal{X}_{0}^{n}+\sum_{i=0}^{k} b\left(a-\mathcal{X}_{i}^{n}\right) \frac{1}{n}+\sum_{i=0}^{k} c \sqrt{\mathcal{X}_{i}^{n}\left(1-\mathcal{X}_{i}^{n}\right)} \frac{\xi_{i+1}^{n}}{\sqrt{n}}
$$

where

$$
\begin{aligned}
E\left(\xi_{k+1}^{n}\right) & =o\left(\frac{1}{n}\right), \\
E\left(\left(\xi_{k+1}^{n}\right)^{2}\right) & =1+o\left(\frac{1}{n}\right), \\
E\left(\left(\xi_{k+1}^{n}\right)^{j}\right) & =o\left(\frac{1}{n}\right), j \geq 3 .
\end{aligned}
$$


We also assume that $\varepsilon_{i+1}^{n}$ in equation (A.1) and $\xi_{i+1}^{n}$ in equation (A.4) are correlated or more precisely that $\varepsilon_{i+1}^{n}=\rho \xi_{i+1}^{n}+\sqrt{1-\rho^{2}} \zeta_{i+1}^{n}$ with $\rho \in(-1,1)$ and $\zeta_{i+1}^{n}$ i.i.d. Gaussian random variables with zero mean, unit variance and uncorrelated with $\xi_{i+1}^{n}$.

If one denotes

$$
\begin{aligned}
W_{k}^{S, n} & :=\frac{1}{\sqrt{n}} \sum_{i=0}^{k} \varepsilon_{i+1}^{n}, \\
W_{k}^{\mathcal{X}, n} & :=\frac{1}{\sqrt{n}} \sum_{i=0}^{k} \xi_{i+1}^{n},
\end{aligned}
$$

one has that:

$$
\left\{\begin{array}{l}
S_{t}^{n}=S_{0}^{n}+\int_{0}^{t} \mu_{\tau}^{n} S_{\tau}^{n} d \tau+\int_{0}^{t} \frac{\sigma}{1-\gamma \mathcal{X}_{\tau}^{n}} S_{\tau}^{n} d W_{\tau}^{S, n} \\
\mathcal{X}_{t}^{n}=\mathcal{X}_{0}^{n}+\int_{0}^{t} b\left(a-\mathcal{X}_{\tau}^{n}\right) d \tau+\int_{0}^{t} c \sqrt{\mathcal{X}_{\tau}^{n}\left(1-\mathcal{X}_{\tau}^{n}\right)} d W_{\tau}^{\mathcal{X}, n}
\end{array}\right.
$$

with $Z_{t}^{n}:=Z_{[n t]}^{n}$ where $Z$ can be any of the random variables that appeared so far in the demonstration.

The result follows from the fact that $\left(W_{t}^{S, n}, W_{t}^{\mathcal{X}, n}\right)$ converges in distribution to a bi-dimensional Brownian motion $\left(W_{t}^{S}, W_{t}^{\mathcal{X}}\right)$ with $d W_{t}^{S} d W_{t}^{\mathcal{X}}=\rho d t$.

Proof of Proposition 2. Let us denote by

$$
\varphi(\tau, \mathcal{X} ; \phi):=E^{Q}\left(\exp \left(i \phi\left(\ln S_{t+\tau}-\ln S_{t}\right)\right) \mid \mathcal{X}_{t}=\mathcal{X}\right)
$$

the characteristic function of $\ln S_{t+\tau}-\ln S_{t} \mid \mathcal{X}_{t}=\mathcal{X}$. One has that

$$
\varphi(\tau, \mathcal{X} ; \phi)=\exp \left(i \phi\left((r-q)-\frac{\sigma^{2}}{2}\right) \tau-\frac{\phi^{2}}{2} \sigma^{2} \tau\right) f(\tau, \mathcal{X} ; \phi)
$$

where the function $f(\tau, \mathcal{X} ; \phi)$ is the solution of the following partial differential equa- 
tion $(\mathrm{PDE})$ :

$$
\begin{aligned}
\frac{\partial f}{\partial \tau} & =\frac{1}{2} c^{2} \mathcal{X}(1-\mathcal{X}) \frac{\partial^{2} f}{\partial \mathcal{X}^{2}}+\left(b^{Q}\left(a^{Q}-\mathcal{X}\right)+(i \phi) \rho \sigma c \frac{\sqrt{\mathcal{X}(1-\mathcal{X})}}{1-\gamma \mathcal{X}}\right) \frac{\partial f}{\partial \mathcal{X}} \\
& +\left[-\frac{i \phi}{2} \sigma^{2}\left(\frac{1}{(1-\gamma x)^{2}}-1\right)+\frac{(i \phi)^{2}}{2} \sigma^{2}\left(\frac{1}{(1-\gamma x)^{2}}-1\right)\right] f
\end{aligned}
$$

with the condition $f(0, \mathcal{X} ; \phi)=1$.

If one employs a formal series expansion for the function $f(\tau, \mathcal{X} ; \phi)$ :

$$
f(\tau, \mathcal{X} ; \phi)=\sum_{n=0}^{\infty} f_{n}(\tau, \mathcal{X}) \frac{(i \phi)^{n}}{n !}
$$

by matching the powers of $(i \phi)$ it follows that $f_{0}(\tau, \mathcal{X}) \equiv 1$ and the functions $f_{n}(\tau, \mathcal{X})$, $n \geq 1$ are the solutions of the following system of PDEs:

$$
\left\{\begin{aligned}
\frac{\partial f_{n}}{\partial \tau} & =\frac{1}{2} c^{2} \mathcal{X}(1-\mathcal{X}) \frac{\partial^{2} f_{n}}{\partial \mathcal{X}^{2}}+b^{Q}\left(a^{Q}-\mathcal{X}\right) \frac{\partial f_{n}}{\partial \mathcal{X}} \\
& -n \frac{\sigma^{2}}{2}\left(\frac{1}{(1-\gamma \mathcal{X})^{2}}-1\right) f_{n-1}+n \rho \sigma c \frac{\sqrt{\mathcal{X}(1-\mathcal{X})}}{1-\gamma \mathcal{X}} \frac{\partial f_{n-1}}{\partial \mathcal{X}} \\
& +n(n-1) \frac{\sigma^{2}}{2}\left(\frac{1}{(1-\gamma \mathcal{X})^{2}}-1\right) f_{n-2}, n \geq 1
\end{aligned}\right.
$$

with the conditions $f_{n}(0, \mathcal{X})=0, n \geq 1$.

The system of PDEs in equation (A.8) can be solved recursively. If one denotes by

$$
\begin{array}{r}
\Phi_{n}(\tau, \mathcal{X})=-n \frac{\sigma^{2}}{2}\left(\frac{1}{(1-\gamma \mathcal{X})^{2}}-1\right) f_{n-1}(\tau, \mathcal{X}) \\
+n \rho \sigma c \frac{\sqrt{\mathcal{X}(1-\mathcal{X})}}{1-\gamma \mathcal{X}} \frac{\partial f_{n-1}}{\partial \mathcal{X}}(\tau, \mathcal{X}) \\
+n(n-1) \frac{\sigma^{2}}{2}\left(\frac{1}{(1-\gamma \mathcal{X})^{2}}-1\right) f_{n-2}(\tau, \mathcal{X})
\end{array}
$$


it follows that

$$
f_{n}(\tau, \mathcal{X})=\int_{0}^{\tau} \int_{0}^{1} \Phi_{n}(t, \varepsilon) G(\mathcal{X}, \tau ; \varepsilon, t) d \varepsilon d t
$$

with the Green function $G(\mathcal{X}, \tau ; \varepsilon, t)$ associated to the PDE

$$
\frac{\partial G}{\partial \tau}=\frac{1}{2} c^{2} \mathcal{X}(1-\mathcal{X}) \frac{\partial^{2} G}{\partial \mathcal{X}^{2}}+b^{Q}\left(a^{Q}-\mathcal{X}\right) \frac{\partial G}{\partial \mathcal{X}}
$$

being given by (e.g. Polyanin, 2001):

$$
G(\mathcal{X}, \tau ; \varepsilon, t)=n\left(\varepsilon ; a^{Q}, b^{Q}, c\right) \sum_{n=0}^{\infty} \exp \left(-\lambda_{n}(\tau-t)\right) P_{n}(\mathcal{X}) P_{n}(\varepsilon)
$$

where $\lambda_{n}=b^{Q} n+\frac{c^{2}}{2} n(n-1)$ are the eigenvalues of the second order differential operator

$$
\frac{1}{2} c^{2} \mathcal{X}(1-\mathcal{X}) \frac{\partial^{2}}{\partial \mathcal{X}^{2}}+b^{Q}\left(a^{Q}-\mathcal{X}\right) \frac{\partial}{\partial \mathcal{X}}
$$

and $P_{n}(\cdot)$ the associated eigenfunctions. These eigenfunctions are the Jacobi polynomials on the interval $(0,1)$. For more details one can consult Gouriéroux, Renault and Valery (2007). The Jacobi polynomials are normalized such that

$$
\int_{0}^{1} P_{i}(x) P_{j}(x) n\left(x ; a^{Q}, b^{Q}, c\right) d x=\delta_{i, j}
$$

where $n\left(x ; a^{Q}, b^{Q}, c\right)$ is the probability distribution function of a Beta distribution with parameters $\alpha=\frac{2 b^{Q}}{c^{2}} a^{Q}$ and $\beta=\frac{2 b^{Q}}{c^{2}}\left(1-a^{Q}\right)$. Inserting the expansion (10) into 
(A.10) and by matching the expansion coefficients one has that

$$
\begin{aligned}
f_{j, m}(\tau)=j \sum_{p=0}^{\infty} g_{m p} \int_{0}^{\tau} e^{-\lambda_{m}(\tau-t)} f_{j-1, p}(t) d t & \\
& +j(j-1) \sum_{p=0}^{\infty} h_{m p} \int_{0}^{\tau} e^{-\lambda_{m}(\tau-t)} f_{j-2, p}(t) d t
\end{aligned}
$$

Therefore, the system of PDEs in (A.8) can be reduced to the system of ODEs in equation (11).

The results about cumulants follow from the relation between the cumulants and the moments of a distribution.

\section{Benchmark Models}

The performance of the Herding model is assessed empirically by comparison to benchmark models: the affine one-factor Heston model and the affine two-factor double Heston model.

The closed form solution of the Heston model is used with the parameters and the FFT implementation as suggested in Moodley (2005), including dividend payments to model European call options:

$$
c\left(S_{t}, V_{t}, K, \tau\right)=S_{t} e^{-q(\tau)} \Pi_{1}\left(\ln \left(S_{t}\right), V_{t}, T, K\right)-K e^{-r(\tau)} \Pi_{2}\left(\ln \left(S_{t}\right), V_{t}, T, K\right)
$$


where $\Pi_{j}$ is consist the characteristic function $f_{\text {Heston }}$ :

$$
\begin{aligned}
\Pi_{j}\left(\ln \left(S_{t}\right), V_{t}, T, K\right) & =\frac{1}{2}+\frac{1}{\pi} \int_{0}^{\infty} \operatorname{Re}\left(\frac{e^{-i x \ln (K)} f_{j}\left(\ln \left(S_{t}\right), V_{t}, T, x\right)}{i x}\right) d x \\
f_{\text {Heston }}\left(\ln \left(S_{t}\right), V_{t}, T, x\right) & =e^{C(\tau, x)+D(\tau, x) V_{t}+i u \ln \left(S_{t}\right)} \\
C(\tau, x) & =\mu x i T+\frac{1}{\sigma^{2}}\left[\left(b_{j}-\rho \sigma x i+d\right)(\tau)-2 \ln \left(\frac{1-g e^{d(\tau)}}{1-g}\right)\right] \\
D(\tau, x) & =\frac{b j-\rho \sigma x i+d}{\sigma^{2}}\left(\frac{1-e^{d(\tau)}}{1-g e^{d(\tau)}}\right) \\
g & =\frac{b j-\rho \sigma x i+d}{b j-\rho \sigma x i-d} \\
d & =\sqrt{(\rho \sigma x i)^{2}-\sigma^{2}\left(2 u_{j} x i-x^{2}\right)}
\end{aligned}
$$

for $\mathrm{j}=1,2$ where: $u_{1}=0.5, u_{2}=-0,5, a=\kappa \theta, b_{1}=\kappa+\lambda-\rho \sigma, b_{2}=\kappa+\lambda$. We approximate the integral in the characteristic function by an n-point Gaussian quadrature rule.

The Heston model is commonly applied by practitioners for its efficiency and improved accuracy in comparison to the classic Black Scholes model.

To broaden the range of used benchmarks we also include the two factor double Heston model developed by Christoffersen, Heston and Jacobs (2009) with the following closed form solution:

$$
\begin{aligned}
C\left(S_{t}, V_{t}, K, \tau\right) & =S_{t} P_{1}-K e^{-r(\tau)} P_{2} \\
P_{1} & =\frac{1}{2}+\frac{1}{\pi} \int_{0}^{\infty} \operatorname{Re}\left(\frac{e^{-i \phi \ln \left(\frac{S(t)}{K}\right)} f\left(V_{1}, V_{2}, \tau, \phi+1\right)}{i \phi}\right) d \phi \\
P_{2} & =\frac{1}{2}+\frac{1}{\pi} \int_{0}^{\infty} \operatorname{Re}\left(\frac{e^{-i \phi \ln \left(\frac{S(t)}{K}\right)} f\left(V_{1}, V_{2}, \tau, \phi\right)}{i \phi}\right) d \phi
\end{aligned}
$$

with the characteristic function being a generalisation of the characteristic func- 
tion of the Heston model:

$$
\begin{aligned}
f\left(V_{1}, V_{2}, \tau, \phi\right) & =r \phi i \tau \frac{a_{1}}{\sigma_{1}^{2}}\left(\left(b_{1}-\rho_{1} \sigma_{1} \phi i+d_{1}\right) \tau-2 \ln \left(\frac{1-g_{1} e^{d_{1} \tau}}{1-g_{1}}\right)\right) \\
& +\frac{a_{2}}{\sigma_{2}^{2}}\left(\left(b_{2}-\rho_{2} \sigma_{2} \phi i+d_{2}\right) \tau-2 \ln \left(\frac{1-g_{2} e^{d_{2} \tau}}{1-g_{2}}\right)\right), \\
B_{j}(\tau, \phi) & =\frac{b j-\rho_{j} \sigma_{j} \phi i+d_{j}}{\sigma_{j}^{2}}\left(\frac{1-e^{d_{j}(\tau)}}{1-g_{j} e^{d_{j}(\tau)}}\right), \\
g_{j} & =\frac{b_{j}-\rho_{j} \sigma_{j} \phi i+d_{j}}{b_{j}-\rho_{j} \sigma_{j} \phi i-d_{j}} \\
d_{j} & =\sqrt{\left(\rho_{j} \sigma_{j} \phi i-b_{j}\right)^{2}+\sigma^{2}\left(\phi i+\phi^{2}\right)}
\end{aligned}
$$

where

$$
\begin{aligned}
& d S=r S d t+\sqrt{V_{1}} S d z_{1}+\sqrt{V_{2}} S d z_{2} \\
& d V_{1}=\left(a_{1}-b_{1} V_{1}\right) d t+\sigma_{1} \sqrt{V_{1}} d z_{3} \\
& d V_{2}=\left(a_{2}-b_{2} V_{2}\right) d t+\sigma_{2} \sqrt{V_{2}} d z_{4}
\end{aligned}
$$

where $\rho_{1}$ is the correlation between $z_{1}$ and $z_{3}$ and $\rho_{2}$ is the correlation between $z_{2}$ and $z_{4}$. Other combinations of $\mathrm{z}$ are uncorrelated. 